\title{
BMJ Open Comorbid insomnia, psychological symptoms and widespread pain among patients suffering from musculoskeletal pain in general practice: a cross- sectional study
}

\author{
Louise Sørensen, ${ }^{1}$ Morten Sig Ager Jensen, ${ }^{1}$ Michael Skovdal Rathleff, ${ }^{1,2}$ \\ Sinead Holden ${ }^{1,2}$
}

To cite: Sørensen L, Jensen MSA, Rathleff MS, et al. Comorbid insomnia, psychological symptoms and widespread pain among patients suffering from musculoskeletal pain in general practice: a cross-sectional study. BMJ Open 2019;9:e031971. doi:10.1136/ bmjopen-2019-031971

- Prepublication history and additional material for this paper are available online. To view please visit the journal (http:// dx.doi.org/10.1136/bmjopen2019-031971).

Received 28 May 2019 Revised 3 June 2019 Accepted 4 June 2019

D) Check for updates

(c) Author(s) (or their employer(s)) 2019. Re-use permitted under CC BY-NC. No commercial re-use. See rights and permissions. Published by BMJ.

${ }^{1}$ Centre for General Practice in Aalborg, Department of Clinical Medicine, Aalborg University, Aalborg, Denmark

${ }^{2} \mathrm{SMI}$, Aalborg Universitet Institut for Medicin og

Sundhedsteknologi, Aalborg, Denmark

Correspondence to

Dr Sinead Holden;

siho@hst.aau.dk

\section{ABSTRACT}

Objective The aim of this study was to examine the association between musculoskeletal (MSK) pain, insomnia, anxiety and depressive symptoms in patients from general practice.

Design This is a cross-sectional study.

Setting This study was conducted in general practice in Denmark.

Participants A consecutive sample of 390 general practice patients (aged 12 years or older) were included; 183 patients with MSK pain and 207 patients without MSK pain. To be included in the MSK pain group, participants with MSK pain were required to report MSK pain at least weekly during the preceding month, which had a negative impact on daily activities.

Primary and secondary outcomes measures The primary outcome was insomnia evaluated by the Athens Insomnia Scale. The secondary outcomes were psychological symptoms assessed by the Hospital Anxiety and Depression Scale.

Results Patients with MSK pain had a significantly higher prevalence of insomnia (difference $25.5 \%, p<0.0001$ ), anxiety (difference $24.3 \%, p<0.0001$ ) and depressive symptoms (difference $11 \%, p<0.0001$ ) compared with patients without MSK pain. Furthermore, patients with MSK pain and comorbid insomnia had significantly higher levels of anxiety and symptoms of depression compared with patients with MSK pain without insomnia $(p<0.0001)$. These relationships remained robust when controlling for age, sex and body mass index in linear regression.

Conclusion One in two patients in general practice report MSK pain. Comorbid MSK pain and insomnia are common and are associated with a higher prevalence of anxiety and depression. This highlights the importance of establishing the presence of insomnia and affective disorders as potentially modifiable factors during treatment of MSK pain in general practice.

\section{INTRODUCTION}

Chronic pain is a global public health concern posing a burden to both the individual and the society. ${ }^{1}$ Of all chronic pain complaints,

\section{Strength and limitations of this study}

- The study was rigorously designed using predefined hypotheses, methods and outcomes that were pilot tested to ensure comprehensibility.

- We used previously validated and reliable questionnaires to assess insomnia and psychological symptoms.

- The limitation of the study is the cross-sectional study design, meaning we cannot make any inferences regarding causality between the parameters assessed. Further longitudinal research is therefore needed to outline the mechanisms, which link the three parameters.

- The self-report nature of the study could lead to an overestimation of some of the problems, for example, the number of patients with clinical anxiety or the number of patients using pain-relieving drugs.

musculoskeletal (MSK) pain is the most common, and the most frequent reason for long-term illness and sick leave in Denmark. ${ }^{2}$ In general practice, MSK pain forms the largest diagnostic group, accounting for $14 \%$ of all consultations and two-thirds of painful conditions. ${ }^{3}$ MSK pain has a large impact as it affects activities of daily living, and patients report lower quality of life compared with pain-free individuals. ${ }^{4}$

Insomnia is a similarly common problem in general practice, affecting $10 \%$ of patients. ${ }^{5}$ Perhaps unsurprisingly, there is a clear association between MSK pain and sleep disturbances. ${ }^{6}$ Patients with MSK pain suffer more from insomnia, and insomnia may be an independent risk factor for developing pain, and is associated with worse prognosis in those with MSK pain. ${ }^{7}$

Psychological factors (such as depression and anxiety) are other important factors 
associated with increased pain and worse prognosis. ${ }^{8}$ Pain may also result in new onset of psychological disorders, suggesting a bidirectional relationship between the two. ${ }^{19}$ Furthermore, these factors may be related to insomnia as there has been a connection between both pain and affective disorders and between affective disorders and insomnia. ${ }^{10}$ Preliminary research from secondary care has found links between chronic pain, mood disorders and insomnia, suggesting a potential association between the three. ${ }^{11-13}$ This is potentially linked to the stress response which is implicated in patients with MSK pain with both mood and sleep disorders. ${ }^{10}$

However, it is unclear whether similar observations can be found in patients with MSK pain in primary care, where most MSK problems are identified and managed. Despite the potential interacting and reciprocal relationships between MSK pain, insomnia and psychological symptoms, there is a lack of research examining all three factors in primary care patients with MSK pain. It remains to be answered if those patients in primary care with comorbid MSK pain and insomnia also have an increased prevalence of psychological symptoms. Given the significant impact of sleep disturbance and affective disorders on MSK pain and the fact that MSK pain is a frequent reason for primary care consultations, the focus of this study was to explore the prevalence, characteristics and links between MSK pain, insomnia, and depressive and anxiety symptoms in primary care patients. This can potentially inform primary care strategies to provide evidence-based multifaceted anticipatory and preventive care for patients with MSK complaints.

The primary aim of this study was to investigate the prevalence of insomnia among patients in general practice with MSK pain compared with control patients in general practice without MSK pain. The hypothesis was that a higher proportion of patients with MSK pain would be classified as 'insomniacs' compared with the control group.

The secondary aim was to explore whether psychological symptoms (depression and anxiety) were higher among patients with MSK pain with concurrent insomnia. The hypothesis was that patients with comorbid MSK pain and insomnia have more psychological symptoms compared with patients with MSK pain alone.

Finally, within the MSK pain group, we aimed to investigate if the prevalence of insomnia and psychological symptoms were more common in patients with widespread MSK pain compared with those with localised pain.

\section{METHODS}

\section{Study design and setting}

This study is designed a cross-sectional questionnaire-based study, conducted between the $6^{\text {th }}-23$ ${ }^{\text {rd }}$ November 2017 in a general practice in Nors, Northern Jutland, Denmark. The general practice has a patient population of 5938 patients (November 2017). The reporting of the manuscript follows Strengthening the Reporting of Observational Studies in Epidemiology guidelines. Prior to conducting the study, a full study protocol was written, in which the outcomes, hypotheses and analyses were predefined (available as online supplementary appendix).

\section{Patient and public involvement}

Patients were not involved with the design of this study.

\section{Participants}

Patients aged 12 years or older consulting the practice during the study period were asked to complete the questionnaire by doctors, nurses or the medical laboratory technician. The participants were not given any information of the content of the questionnaire beforehand, thus reducing the risk of selection bias.

\section{Measures}

The self-report questionnaire was based on existing and validated surveys, and consisted of demographic parameters, self-reported MSK pain, insomnia, anxiety and depressive symptoms (outlined below). Prior to data collection, the questionnaire was piloted in the clinic on 18 patients and adolescents aged 12-14 years to ensure comprehensibility. The same questionnaire was handed out to children and adults.

\section{Musculoskeletal Pain}

A structured pain questionnaire was designed to assess different aspects of MSK pain. Patients were asked if they had experienced pain at least weekly during the preceding month, and if so, to mark the exact location of their pain on an outlined manikin of the human body. Those reporting pain were subsequently asked to report pain frequency (rarely, monthly, once a week, more than once a week, almost daily), pain duration and pain intensity (measured as average and worst pain during the preceding week on numeric rating scale ranging from 0 to 10 ranging from no pain to worst imaginable pain). Patients were asked to indicate if they had consulted their general practitioner for their pain, and if the cause of pain was known (eg, a specific diagnosis such as osteoarthritis). They were also asked if pain had a negative influence on their daily activities (none, little, moderate, large or cannot perform due to pain) and whether they took any pain-relieving medication.

Participants were eligible for inclusion in the MSK pain group if they met the following criteria:

1. MSK pain at least once a week during the preceding month.

2. This pain negatively interfered with their usual activities.

Participants were categorised as having widespread pain (WSP) if they fulfilled the criteria from The American College of Rheumatology: pain in both the left and the right sides of the body, pain above and below the waist, and pain in the axial skeleton. ${ }^{14}$ 


\section{Sleep}

The primary outcome was the Athens Insomnia Scale (AIS-8). ${ }^{15}$ It consists of eight items scored on Likerttype scales ranging from 0 (no problem at all) to 3 (very severe sleep problem). The patients rated the item positive if it occurred at least three times per week during the last month. The internal consistency and external validity of the AIS- 8 are high (Cronbach's alpha $=0.89$ and Pearson's correlation coefficient with the Sleep Problems Scale $=0.90$, respectively) and it has established testretest reliability $(0.89) .^{15}$

The sleep questionnaire was translated using the dualpanel translation method (translation and use of the AIS-8 by permission of Professor C. R. Soldatos, author of AIS-8). ${ }^{15}$ The cut-off score for classifying insomnia is $6 .{ }^{16}$

\section{Psychological symptoms}

Psychological symptoms were anxiety and depressive symptoms, assessed by the Hospital Anxiety and Depression Scale (HADS). HADS is a 14-item self-report questionnaire, which consists of an anxiety (HADS-A) and a depression subscale (HADS-D). Responses are scored from 0 to 3 (each subscale ranges from 0 to 21).

Cronbach's alpha is 0.83 for HADS-A and 0.82 for HADS-D, and the validity of HADS is good to very good (correlation 0.60-0.80) compared with Beck's Depression Inventory. ${ }^{17}$ HADS has previously been translated into Danish using the forward-backward translation method, and the translated version has been cross-culturally validated. $^{18}$

\section{Statistical methods}

\section{Sample size calculation}

Based on data from Paparrigopoulos et al, ${ }^{19}$ the proportion of participants with chronic disease with insomnia (according to the AIS-8) was $42.8 \%$, compared with $15.5 \%$ of controls. We estimated that $20 \%$ of the patients in our control group would be classified as having insomnia (as our sample was general practice patients, and some may suffer from chronic diseases), and $35 \%$ of the patients from our MSK pain group would be classified as having insomnia. Using a p value of 0.05 and power of $80 \%$, the ratio between the sample sizes was set at 0.14 (as MSK pain accounts for $14 \%$ of all general practice consultations $\left.^{3}\right)$. This gave us a final sample size of 73 patients with MSK pain, from an estimated total of 598 patients.

\section{Statistical analyses}

All data from the questionnaires were entered electronically and subsequently rechecked for typing errors by one person. Missing data in HADS were managed using the 'half rule' method, meaning that the patient's subscale mean (for the HADS-A and HADS-D, respectively) was used to fill in the missing data, given that at least half of the subscale was answered; otherwise, the patient was excluded. ${ }^{20}$ The same method was used for the AIS- 8 . Patients were excluded from the study if they had failed to answer the questions, which allocated them to either the MSK pain group or the control group.

$\chi^{2}$ test of independence was used for the primary analysis to determine differences in proportions of those categorised as 'insomniacs' in the MSK pain group versus control group. Unpaired t-tests were used to analyse continuous scores from HADS, if normally distributed (checked by visual assessment of $\mathrm{Q}-\mathrm{Q}$ plots), to detect differences in anxiety and depressive symptoms between the MSK pain group versus control group and reported using mean values and effect sizes. Unpaired t-tests were used to determine differences between subgroups (patients with MSK pain alone vs patients with MSK pain and comorbid insomnia, and patients with localised MSK pain vs WSP).

$\chi^{2}$ test of independence was applied to detect differences in proportion of insomniacs (categorised according to the AIS-8 cut score of 6 ) between patients with localised pain versus WSP. For all analyses, the level of statistical significance was set at $\mathrm{p}<0.05$. Data were analysed using the SPSS software (Version 22.0).

\section{Post hoc regression analyses}

Based on peer review comments, post hoc regression analyses were run to ensure the reported relationships were not an artefact due to differences in age, sex and body mass index (BMI). For the primary outcome (sleep problems measured on the AIS-8 as a continuous variable), hierarchical linear regression was conducted to test the added predictive value of MSK pain (pain at least once a week during the preceding month and this pain negatively interfered with usual activities) in predicting sleep problems when adjusting for age, BMI and sex (male vs female). Therefore, block 1 included the age, sex and BMI, and block 2 included MSK pain as a dichotomous predictor variable.

This was also done for the secondary outcomes depression (HADS-D) and separately anxiety (HADS-A). For the secondary outcomes, age, sex and BMI were included in block 1 to evaluate the additional predictive capacity of MSK pain in block 2, and insomnia (as a binary predictor variable based on AIS cut score) in block 3 .

\section{RESULTS}

A total of 424 patients out of 977 eligible patients $(43.4 \%)$ participated in the study. Of the subjects who responded, 34 were excluded due to incomplete data (in either the AIS-8, HADS or the pain questionnaire). Consequently, 390 patients were included in the study, of which 183 $(46.9 \%)$ were categorised as patients with MSK pain (mean age $54.2 \pm 18.5$ years) and 207 (53.1\%) controls (mean age $53.7 \pm 21.0$ years).

Demographic characteristics are presented in table 1. Of those with MSK pain, $71 \%$ had daily pain and $58 \%$ had contacted their general practitioner (GP) for their pain complaint (table 2). 


\begin{tabular}{|c|c|c|}
\hline & MSK pain group & $\begin{array}{l}\text { Control } \\
\text { group }\end{array}$ \\
\hline Patients, n (\%) & $183(46.9)$ & 207 (53.1) \\
\hline \multicolumn{3}{|l|}{ Sex, n (\%) } \\
\hline Male & $63(34.4)$ & $93(44.9)$ \\
\hline Female & $120(65.6)$ & $114(55.1)$ \\
\hline \multicolumn{3}{|l|}{ Age in years, $n(\%)$} \\
\hline $12-19$ & $9(4.9)$ & $22(10.8)$ \\
\hline 20-29 & $15(8.2)$ & 18 (8.9) \\
\hline 30-39 & $12(6.6)$ & $17(8.4)$ \\
\hline $40-49$ & $31(17.0)$ & 20 (9.9) \\
\hline $50-59$ & 33 (18.1) & $23(11.3)$ \\
\hline $60-69$ & $41(22.5)$ & $44(21.7)$ \\
\hline $70-79$ & $30(16.5)$ & $46(22.7)$ \\
\hline 80 and older & $11(6.0)$ & $13(6.4)$ \\
\hline BMI, mean (SD) & $27.9(5.9)$ & $26.4(5.0)$ \\
\hline
\end{tabular}

There are four missing values for age in the control group and one missing value in the musculoskeletal (MSK) pain group.

There are 16 missing values for body mass index (BMI) in

the control group and 6 missing values in the MSK pain group.

\section{Prevalence of insomnia}

A significantly greater proportion of the MSK pain group had insomnia compared with the control group $(65.6 \%$

\section{Table 2 Pain characteristics (MSK pain group)}

MSK pain

group $(n=183)$

\begin{tabular}{|c|c|}
\hline \multicolumn{2}{|l|}{ Pain frequency, $\mathrm{n}(\%)$} \\
\hline Once a week & $10(5.5)$ \\
\hline Several times a week & $43(23.6)$ \\
\hline Almost daily & $129(70.9)$ \\
\hline $\begin{array}{l}\text { Average pain (numeric rating scale, mean } \\
\text { (SD) }\end{array}$ & $5.6(1.7)$ \\
\hline $\begin{array}{l}\text { Duration of symptoms (months), median } \\
\text { (IQR) }\end{array}$ & $24(9-85)$ \\
\hline \multicolumn{2}{|l|}{ Use of pain-relieving drugs, $\mathrm{n}(\%)$} \\
\hline Yes & $133(73.5)$ \\
\hline No & $48(26.5)$ \\
\hline \multicolumn{2}{|c|}{ Contacts to GP for pain during the previous 6 months, $n(\%)$} \\
\hline Several contacts & $55(30.1)$ \\
\hline One contact & $51(27.9)$ \\
\hline No contacts & $77(42.1)$ \\
\hline
\end{tabular}

There is 1 missing value for pain frequency, 4 missing values for numeric rating scale, 45 missing values for duration of symptoms, 2 missing values for use of pain-relieving drugs and 0 missing value for number of contacts to GP.

MSK, musculoskeletal. vs $40.1 \%$; mean difference 25.5 (95\% CI 15.6 to 34.6 ); $\chi^{2}=25.26 ; \mathrm{p}<0.0001 ;$ table 3 ).

\section{Anxiety and depressive symptoms}

The MSK pain group had significantly higher levels of anxiety as illustrated by higher HADS-A score compared with the control group (mean difference 2.6 (95\% CI 1.8 to 3.4 ); t-value $=6.718$; $\mathrm{p}<0.0001$; table 3 ). Similarly, HADS-D was significantly higher in the MSK pain group compared with the controls (mean difference $1.6(95 \%$ CI 1.0 to 2.2); t-value $=5.346$; $\mathrm{p}<0.0001$; table 3 ).

\section{Subgroup comparisons}

Patients with MSK pain and comorbid insomnia had significantly greater anxiety and depressive symptoms compared with patients with MSK pain and no insomnia (mean difference 4.1 (95\% CI 2.9 to 5.2); $\mathrm{t}$-value $=6.871$; $\mathrm{p}<0.0001$ and mean difference 3.2 (95\% CI 2.3 to 4.2 ); $\mathrm{t}$-value $=6.716 ; \mathrm{p}<0.0001$, respectively; table 4 ).

A significantly greater proportion of patients with WSP had insomnia (AIS-8 score $\geq 6$ ) compared with patients with localised pain $(78.0 \%$ vs $59.7 \%$; difference in proportions 18.3 (95\% CI 3.7 to 30.7); $\mathrm{p}<0.05$; table 5). Patients with WSP also had significantly more depressive symptoms compared with patients with localised MSK pain (mean difference 1.2 (95\% CI 0.1 to 2.3); $\mathrm{t}$-value $=2.212 ; \mathrm{p}<0.05$; table 5). There were no statistical differences in anxiety (as measured by HADS-A) score between the two groups (mean difference (95\% CI -0.7 to 1.9 ); $\mathrm{t}$-value $=0.893$; $\mathrm{p}=0.373$; table 5$)$.

\section{Regression models adjusting for sex, age and BMI}

For the outcome of sleep problems (AIS-8), the hierarchical linear regression was statistically significant, with the final model yielding an $R^{2}=0.191, F(4,360)=21.3$; $\mathrm{p}<0.001$. The addition of MSK pain to the model (block 2) led to a statistically significant increase in $R^{2}=0.132$, $F(1,360)=58.96 ; p<0.0001$. See online supplementary file for full details.

For the secondary outcomes of depression and anxiety, the final models were statistically significant (depression $\mathrm{R}^{2}=0.388, \mathrm{~F}(5,359)=29.08 ; \mathrm{p}<0.001$ ) (anxiety $\left.\mathrm{R}^{2}=0.539, \quad \mathrm{~F}(5,359)=29.45 ; \mathrm{p}<0.001\right)$. The addition of MSK pain significantly improved the model compared with block 1 for depressive symptoms $\left(\mathrm{R}^{2}\right.$ change $\left.=0.06 ; \mathrm{F}(1,360)=24.52 ; \mathrm{p}<0.001\right)$ and anxiety $\left(\mathrm{R}^{2}\right.$ change $\left.=0.088, \mathrm{~F}(1,360)=37.15 ; \mathrm{p}<0.001\right)$ subscales. The third block with the addition of insomnia significantly improved the model for depressive $\left(R^{2}\right.$ change $=0.168, \mathrm{~F}(1,359)=84.93 ; \mathrm{p}<0.001)$ and anxiety $\left(\mathrm{R}^{2}\right.$ change $\left.=0.144, \mathrm{~F}(1,359)=72.93 ; \mathrm{p}<0.001\right)$ symptoms (see online supplementary file for full model details).

This underlines that MSK accounts for a significant proportion of the variance in predicting sleep problems, anxiety and depressive symptoms, and that insomnia has further predictive capacity of psychological symptoms, independent of MSK pain. 
Table 3 Sleep and psychological characteristics

\begin{tabular}{|c|c|c|c|c|c|}
\hline & $\begin{array}{l}\text { MSK pain group } \\
(n=183)\end{array}$ & $\begin{array}{l}\text { Control group } \\
(n=207)\end{array}$ & $\begin{array}{l}\text { Difference* } \\
(95 \% \mathrm{Cl})\end{array}$ & $\begin{array}{l}\text { Effect size } \\
\text { (Cohen's } d \text { ) }\end{array}$ & P value $\dagger$ \\
\hline \multicolumn{6}{|l|}{ Sleep, n (\%) } \\
\hline Insomnia (AIS-8 score $\geq 6$ ) & $120(65.6)$ & $83(40.1)$ & 25.5 (15.6 to 34.6$)$ & $\mathrm{N} / \mathrm{A}$ & $<0.0001$ \\
\hline \multicolumn{6}{|l|}{ Sleep, mean (SD) } \\
\hline AIS-8 (0-24) & $9.1(5.7)$ & $5.1(4.1)$ & 4.0 (3.0 to 5.0$)$ & 0.81 & $<0.0001$ \\
\hline \multicolumn{6}{|l|}{ Anxiety and depression, $\mathrm{n}(\%)$} \\
\hline Anxiety (HADS-A $\geq 8$ ) & $71(38.8)$ & $30(14.5)$ & 24.3 (15.6 to 32.6 ) & & $<0.0001$ \\
\hline Depression (HADS-D $\geq 8$ ) & $28(15.3)$ & $9(4.3)$ & $11.0(5.1$ to 17.2$)$ & N/A & $<0.0001$ \\
\hline \multicolumn{6}{|c|}{ Anxiety and depression, mean (SD) } \\
\hline HADS-A (0-21) & $6.5(4.3)$ & $3.9(3.4)$ & 2.6 (1.8 to 3.4$)$ & 0.67 & $<0.0001$ \\
\hline HADS-D (0-21) & $3.9(3.5)$ & $2.3(2.4)$ & $1.6(1.0$ to 2.2$)$ & 0.53 & $<0.0001$ \\
\hline
\end{tabular}

${ }^{*}$ Indicates difference in proportions with $95 \% \mathrm{Cl}$ for categorical variables and mean difference with $95 \% \mathrm{Cl}$ for continuous data, respectively. †Statistically significant values are highlighted in bold. Unpaired t-tests were used to determine $\mathrm{p}$ values for continuous data, whereas $\chi^{2}$ tests were used to determine $p$ values for difference in proportions (categorical variables).

AIS-8, Athens Insomnia Scale; HADS-A, Hospital Anxiety and Depression Scale-Anxiety; HADS-D, Hospital Anxiety and Depression ScaleDepression; MSK, musculoskeletal.

\section{DISCUSSION}

\section{Main findings}

The findings of this study indicate that the typical patient in general practice with MSK pain commonly experiences co-occurring insomnia, anxiety and depressive symptoms. Regression analyses confirmed the independent associations, after adjusting for age, sex and BMI, with MSK contributing to an addition of $13.2 \%$ of the explain variance in sleep problems, $6.0 \%$ in depressive symptoms and $8.8 \%$ in anxiety. Insomnia further significantly improved the prediction of psychological symptoms greater than MSK pain alone.

Importantly, patients who suffer from comorbid MSK pain and insomnia make up a high proportion of patients with MSK pain and will have more severe symptoms of depression and anxiety compared with those only suffering from MSK pain. Our results document a complex relationship between MSK pain, insomnia, anxiety and depression. These factors may depend on the extent of the pain, as those with WSP were more likely to experience insomnia and had more severe depressive symptoms. This highlights the importance of GPs to be cognisant to consider patients' pain complaints in the light of the biopsychosocial model. The high prevalence of both insomnia and WSP underscores the need for general practitioners to consider and enquire about these factors when treating patients with MSK pain in general practice.

\section{Comparison with previous studies}

The high prevalence of MSK pain and coexisting insomnia, depressive symptoms and anxiety in general practice may be partially attributable for MSK complaints being a leading cause for years lived with disability, globally. ${ }^{1}$ Our findings document a strong link between some of the conditions that account for the highest number of years lived with disability; MSK disorders and psychological symptoms. These common conditions rarely exist in isolation. Our findings are in line with research in patients with chronic low back pain, documenting poorer self-reported sleep, compared with individuals without pain, ${ }^{21}$ and showing impaired sleep in patients when using objective measures of sleep. ${ }^{22}$

Table 4 Subgroup comparisons on sleep and psychological characteristics between patients with MSK pain and comorbid insomnia and patients with MSK pain with no insomnia

\begin{tabular}{|c|c|c|c|c|c|}
\hline & $\begin{array}{l}\text { MSK pain patients } \\
\text { with clinical insomnia } \\
(n=120)\end{array}$ & $\begin{array}{l}\text { MSK pain patients } \\
\text { with no clinical } \\
\text { insomnia }(n=63)\end{array}$ & $\begin{array}{l}\text { Mean difference } \\
(95 \% \mathrm{Cl})\end{array}$ & $\begin{array}{l}\text { Effect size } \\
\text { (Cohen's d) }\end{array}$ & P value* \\
\hline \multicolumn{6}{|c|}{ Anxiety and depression, mean (SD) } \\
\hline HADS-A (0-21) & $7.9(4.2)$ & $3.9(2.8)$ & $4.1(2.9-5.2)$ & 1.12 & $<0.0001$ \\
\hline HADS-D (0-21) & $5.0(3.6)$ & $1.8(1.9)$ & $3.2(2.3-4.2)$ & 1.11 & $<0.0001$ \\
\hline
\end{tabular}

*Statistically significant values are highlighted in bold. Unpaired t-tests were used to determine $p$ values.

HADS-A, Hospital Anxiety and Depression Scale-Anxiety; HADS-D, Hospital Anxiety and Depression Scale-Depression;

MSK, musculoskeletal. 
Table 5 Subgroup comparisons on sleep and psychological characteristics between patients with localised MSK pain and WSP

\begin{tabular}{|c|c|c|c|c|c|}
\hline & $\begin{array}{l}\text { Localised MSK } \\
\text { pain }(n=124)\end{array}$ & $\begin{array}{l}\text { WSP } \\
(n=59)\end{array}$ & $\begin{array}{l}\text { Difference* }^{*} \\
(95 \% \mathrm{Cl})\end{array}$ & $\begin{array}{l}\text { Effect size } \\
\text { (Cohen's } d \text { ) }\end{array}$ & P value \\
\hline Insomnia (AIS-8 score $\geq 6$ ), n (\%) & $74(59.7)$ & $46(78.0)$ & 18.3 (3.7 to 30.7$)$ & N/A & 0.015 \\
\hline HADS-A (0-21) & $6.4(4.2)$ & $6.9(4.4)$ & $0.6(-0.7$ to 1.9$)$ & 0.12 & 0.373 \\
\hline HADS-D (0-21) & $3.5(3.3)$ & $4.7(3.8)$ & 1.2 (0.1 to 2.3$)$ & 0.34 & 0.028 \\
\hline
\end{tabular}

*Indicates difference in proportions with $95 \% \mathrm{CI}$ for AIS-8 and mean difference with 95\% CI for HADS.

†Statistically significant values are highlighted in bold. All $p$ values for HADS were determined by unpaired t-tests, whereas the $p$ value for AIS- 8 was determined by $\chi^{2}$ test.

AIS-8, Athens Insomnia Scale; HADS-A, Hospital Anxiety and Depression Scale-Anxiety; HADS-D, Hospital Anxiety and Depression ScaleDepression; MSK, musculoskeletal; WSP, widespread pain.

However, until now there has been a complete dearth of knowledge on the coexistence and associations in patients from general practice, with this study being the first to document the prevalence and coexistence of all three parameters.

The patients with MSK in our study scored 9.1 on the AIS-8, suggesting they suffer from quite severe insomnia, as this is notably worse compared with patients with rheumatoid arthritis (a disease with predominantly musculoskeletal manifestations), who report a mean score of 6.8. ${ }^{23}$ This underscores the magnitude of insomnia in this population from general practice and may be due to the high severity of pain complaints in our sample.

A longitudinal study in five UK general practices found a prevalence of insomnia of $37 \%$, which is comparable to the current study ( $40 \%$ in those without MSK pain). Further, they demonstrated that insomnia was associated with pre-existing pain, anxiety and depression, and insomnia was a risk factor for the subsequent onset of anxiety, depression and pain. ${ }^{24}$ This underpins the complex bidirectional relationship between the factors, and the need to both consider insomnia in those presenting with pain and also pain problems in those presenting to the GP for insomnia. This is particularly important as not all of our sample reported having consulted the GP specifically for the MSK pain complaint.

With nearly $80 \%$ of those with WSP meeting the threshold for insomnia, our findings are consistent with previous studies showing that insomnia is more prevalent in patients with pain in more than one region of the body. ${ }^{25}$ Patients with WSP similarly have more severe symptoms of depression and anxiety compared with patients with localised pain. ${ }^{26}$ Similarly, in our sample, we documented significantly higher levels of depression among patients with WSP.

\section{Explanation of findings; the causal link between pain, anxiety, depression and sleep}

There is a growing body of research suggesting possible common pathophysiological and behavioural mechanisms between chronic pain, depressive/mood disorders and insomnia. ${ }^{727}$ One explanation is the stress response system as it plays a key role in the relationship between insomnia and affective disorders in patients with MSK pain. ${ }^{10}$ Tissue injuries may lead to the downregulation of the serotonergic pathway, as well as the release and upregulation of key chemical mediators such as proinflammatory cytokines that may lead to the onset of depression and insomnia. ${ }^{27}$ Sleep deprivation has also been linked to increased pain perception and hyperalgesia ${ }^{28}$ and altered ability to modulate pain, ${ }^{29}$ which may explain why insomnia could lead to the development or prognosis of chronic pain. Furthermore, the physiological and psychological consequences of sleep problems could impact an individual's ability to cope with pain particularly as sleep efficiency has been related to activity limitations and health-related quality of life in adolescents with chronic pain. ${ }^{30}$ It is likely that there is a complex cyclical relationship between the three, which could be addressed during treatment to enable improved patient care.

\section{Implications for general practice and research}

While determining causality is difficult, research consistently indicates that insomnia may both be a risk factor for the onset of pain and contribute to the development of chronic pain, ${ }^{7}$ highlighting the importance of sleep. Comorbid complaints may increase the risk of poor prognosis $^{8}$ and improvements in depression (eg, antidepressants or psychotherapy interventions), ${ }^{31}$ anxiety and sleep $^{32}$ (eg, by cognitive behavioural therapy) have all been associated with improvements in pain, highlighting the need for including these aspects during the clinical consultation. While sleep disorders may be an independent risk factor for onset of pain, they also represent an important modifiable factor. For example, in adolescents with chronic pain, greater number of minutes awake following sleep onset is associated with higher pain the following day. ${ }^{33}$ In general practice, it is useful to know which treatment targets are likely to lead to improvements in a patient's symptoms. GPs should be aware of the high prevalence of comorbid psychological symptoms found in this cohort of patients from general practice, particularly considering the association between improvements 
in psychological problems/insomniaand improvements in pain. ${ }^{31} 32$

Although robust evidence to demonstrate effectiveness of treatments for these comorbid conditions is still needed to confidently recommend screening, this study reiterates the need for a GP to be aware that among primary care attendees consulting for MSK pain, many are likely to present with coexisting mood and sleep disturbance symptoms. Furthermore, with the view to recognise unidentified MSK pain, a GP may choose to explore the presence of associated MSK pain in patients presenting with insomnia.

\section{Strengths and limitations}

The study was rigorously designed using predefined hypotheses, methods and outcomes that were pilot tested to ensure comprehensibility. We used previously validated and reliable questionnaires to assess insomnia and psychological symptoms.

The limitation of the study is the cross-sectional study design, meaning we cannot make any inferences regarding causality between the parameters assessed. Despite having no data on non-respondents, or non-completed responses, the threat of selection bias is low due to potential participants not being aware of the content of the questionnaire. Further longitudinal research is needed to outline the mechanisms, which link the three parameters. The self-report nature of the study could lead to an overestimation of some of the problems, for example, the number of patients with clinical anxiety or the number of patients using pain-relieving drugs.

\section{Future directions}

With increasing global healthcare costs resulting from the comorbidity of these four health conditions (MSK pain, anxiety, depression and insomnia), there is the need to define to further understand how we conceptualise and treat these symptoms in general practice. Cognitive behavioural therapy targeted at improving sleep has been effective in reducing sleep disturbances and has a positive effect on pain in older adults with osteoarthritis. ${ }^{32}$ This research could be extended to patients with MSK pain in general practice to determine if this can also positively influence psychological symptoms which commonly occur in conjunction with comorbid insomnia and pain problems. Further, we need to understand the development of MSK pain and coexisting psychological symptoms to understand if there are critical time-points in which GPs can positively affect this trajectory.

\section{CONCLUSION}

One in two patients from general practice report MSK pain which interferes with activities of daily living. The patients with MSK pain more commonly suffered from insomnia and had depressive and anxiety symptoms. Patients who suffered from both MSK pain and insomnia experienced depressive symptoms and anxiety far more than patients only suffering from pain. Insomnia and affective disorders might be potentially modifiable factors to target as part of the treatment for MSK pain in general practice.

Contributors LS, MSR, MSAJ and SH all participated in the conception and design of the study and drafting of the study protocol. LS and MSAJ were responsible for the collection of data. LS and SH conducted the analyses, and all authors took part in the interpretation of the results. All authors critically revised the manuscript for important intellectual content and approved the final version of the manuscript.

Funding The project was internally funded by the Research Unit for General Practice in Aalborg.

Competing interests None declared.

Patient consent for publication Not required.

Ethics approval This questionnaire-based studies was exempt from approval by the research ethics committee according to Danish law. It was voluntary to participate in the study and all personal data were securely stored by permission of Datatilsynet (ProjectID: 2017-215).

Provenance and peer review Not commissioned; externally peer reviewed.

Data sharing statement Data are available on reasonable request.

Open access This is an open access article distributed in accordance with the Creative Commons Attribution Non Commercial (CC BY-NC 4.0) license, which permits others to distribute, remix, adapt, build upon this work non-commercially, and license their derivative works on different terms, provided the original work is properly cited, appropriate credit is given, any changes made indicated, and the use is non-commercial. See: http://creativecommons.org/licenses/by-nc/4.0/.

\section{REFERENCES}

1. GBD 2016 Disease and Injury Incidence and Prevalence Collaborators. Global, regional, and national incidence, prevalence, and years lived with disability for 328 diseases and injuries for 195 countries, 1990-2016: a systematic analysis for the Global Burden of Disease Study 2016. Lancet 2017;390:1211-59.

2. Flaschs EM, Eriksen L, Koch MB, et al. Sygdomsbyrden i Danmark sygdomme. København: Sundhedsstyrelsen, 2015:384.

3. Jordan KP, Kadam UT, Hayward R, et al. Annual consultation prevalence of regional musculoskeletal problems in primary care: an observational study. BMC Musculoskelet Disord 2010;11:144.

4. Breivik $H$, Collett $B$, Ventafridda $V$, et al. Survey of chronic pain in Europe: prevalence, impact on daily life, and treatment. Eur J Pain 2006;10:287-333.

5. Simon GE, VonKorff M. Prevalence, burden, and treatment of insomnia in primary care. Am J Psychiatry 1997;154:1417-23.

6. Choy $\mathrm{EH}$. The role of sleep in pain and fibromyalgia. Nat Rev Rheumatol 2015;11:513-20.

7. Finan PH, Goodin BR, Smith MT. The association of sleep and pain: an update and a path forward. J Pain 2013;14:1539-52.

8. Jansson-Fröjmark M, Boersma K. Bidirectionality between pain and insomnia symptoms: a prospective study. Br J Health Psychol 2012;17:420-31.

9. Gureje O, Simon GE, Von Korff M. A cross-national study of the course of persistent pain in primary care. Pain 2001;92(1-2):195-200.

10. Palagini L, Palagini L, Carmassi C, et al. Transdiagnostic factors across fibromyalgia and mental disorders: sleep disturbances may play a key role. A clinical review. Clin Exp Rheumatol 2016;34:28-31.

11. O'Brien EM, Waxenberg LB, Atchison JW, et al. Negative mood mediates the effect of poor sleep on pain among chronic pain patients. Clin J Pain 2010;26:310-9.

12. Emery PC, Wilson KG, Kowal J. Major depressive disorder and sleep disturbance in patients with chronic pain. Pain Res Manag 2014;19:35-41.

13. Miró E, Martínez MP, Sánchez Al, et al. When is pain related to emotional distress and daily functioning in fibromyalgia syndrome? The mediating roles of self-efficacy and sleep quality. $\mathrm{Br} \mathrm{J}$ Health Psychol 2011;16:799-814.

14. Wolfe F, Smythe HA, Yunus MB, et al. The American College of Rheumatology 1990 Criteria for the Classification of Fibromyalgia. 
Report of the Multicenter Criteria Committee. Arthritis Rheum 1990;33:160-72.

15. Soldatos CR, Dikeos DG, Paparrigopoulos TJ. Athens Insomnia Scale: validation of an instrument based on ICD-10 criteria. $J$ Psychosom Res 2000;48:555-60.

16. Soldatos CR, Dikeos DG, Paparrigopoulos TJ. The diagnostic validity of the Athens Insomnia Scale. J Psychosom Res 2003;55:263-7.

17. Bjelland I, Dahl AA, Haug TT, et al. The validity of the Hospital Anxiety and Depression Scale. An updated literature review. J Psychosom Res 2002;52:69-77.

18. Hospital Anxiety and Depression Scale (HADS) [Danish], 1994.

19. Paparrigopoulos T, Tzavara C, Theleritis $C$, et al. Insomnia and its correlates in a representative sample of the Greek population. BMC Public Health 2010;10:531.

20. Bell ML, Fairclough DL, Fiero MH, et al. Handling missing items in the Hospital Anxiety and Depression Scale (HADS): a simulation study. BMC Res Notes 2016;9:479.

21. O'Donoghue GM, Fox N, Heneghan C, et al. Objective and subjective assessment of sleep in chronic low back pain patients compared with healthy age and gender matched controls: a pilot study. BMC Musculoskelet Disord 2009;10:122.

22. Olsen MN, Sherry DD, Boyne K, et al. Relationship between sleep and pain in adolescents with juvenile primary fibromyalgia syndrome. Sleep 2013;36:509-16.

23. Westhovens R, Van der Elst K, Matthys A, et al. Sleep problems in patients with rheumatoid arthritis. J Rheumatol 2014;41:31-40.

24. Morphy H, Dunn KM, Lewis M, et al. Epidemiology of insomnia: a longitudinal study in a UK population. Sleep 2007;30:274-80.
25. Tang NK, McBeth J, Jordan KP, et al. Impact of musculoskeletal pain on insomnia onset: a prospective cohort study. Rheumatology 2015;54:248-56.

26. Alföldi P, Dragioti E, Wiklund T, et al. Spreading of pain and insomnia in patients with chronic pain: results from a national quality registry (SQRP). J Rehabil Med 2017;49:63-70.

27. Boakye PA, Olechowski C, Rashiq S, et al. A Critical Review of Neurobiological Factors Involved in the Interactions Between Chronic Pain, Depression, and Sleep Disruption. Clin J Pain 2016;32:327-36.

28. Lautenbacher S, Kundermann B, Krieg JC. Sleep deprivation and pain perception. Sleep Med Rev 2006;10:357-69.

29. Haack M, Scott-Sutherland J, Santangelo G, et al. Pain sensitivity and modulation in primary insomnia. Eur J Pain 2012;16:522-33.

30. Palermo TM, Fonareva I, Janosy NR. Sleep quality and efficiency in adolescents with chronic pain: relationship with activity limitations and health-related quality of life. Behav Sleep Med 2008;6:234-50.

31. Lin EH, Katon W, Von Korff M, et al. Effect of improving depression care on pain and functional outcomes among older adults with arthritis: a randomized controlled trial. JAMA 2003;290:2428-9.

32. Vitiello MV, Rybarczyk B, Von Korff M, et al. Cognitive behavioral therapy for insomnia improves sleep and decreases pain in older adults with co-morbid insomnia and osteoarthritis. J Clin Sleep Med 2009;5:355-62.

33. Lewandowski AS, Palermo TM, De la Motte S, et al. Temporal daily associations between pain and sleep in adolescents with chronic pain versus healthy adolescents. Pain 2010;151:220-5. 